\title{
EFFECT OF VARIATION OF ANGLES OF TERNARY LINKS ON THE GAIT PATTERN OF KLANN'S MECHANISM
}

\author{
Pratik Walimbe $^{1}$, Pranavkumar Nehrotra ${ }^{2}$, Akash Gunjal ${ }^{3}$, Manish Chaudhari ${ }^{4}$ \\ ${ }^{I}$ Student, Department of Mechanical Engineering, Vishwakarma Institute of Technology, Maharashtra, India \\ ${ }^{2}$ Student, Department of Mechanical Engineering, Vishwakarma Institute of Technology, Maharashtra, India \\ ${ }^{3}$ Student, Department of Mechanical Engineering, Vishwakarma Institute of Technology, Maharashtra, India \\ ${ }^{4}$ Student, Department of Mechanical Engineering, Vishwakarma Institute of Technology, Maharashtra, India
}

\begin{abstract}
Although wheeled robots have been an area of interest for researchers, the major drawback with the wheels is that, as the ground gets rougher and rougher, wheels tend to become insubstantial i.e. less and less effective. But the solution to this predicament already exists. Thanks to the nature's solution which has no such problems. The solution is 'legs'. The idea of replicating a natural concept into an artificial product has led the foundations of the newlyevolved branch of science and engineering called 'Biomimetics' or also known as 'Biomimicry'. The underlying intent of this 'Biomimetics' or 'Biomimicry' based project is is to study the effect of variation of angles of ternary links on gaitpattern of an octopod robot using Klann's linkage/Klann's mechanism. For the replication of the leg of an insect, the use of Klann's linkage has been demonstrated. The mathematical modelling and mathematical formulation for the Klann's linkage has been stated by using 'Complex Algebra Method'. The position analysis using Complex Algebra Method enables us to find the nature of gait pattern of the Klann's mechanism. Then effect of variation of angles of couplerlinksonthe gait pattern of Klann's mechanism has been simulated. Through the analysis and simulation process, a number of possible solutions were obtained. But a careful analysis will lead to the conclusion thatthemost precise i.e. optimum gait pattern is obtained by maintaining a very particular combination of angles of coupler links.Thus, through a rigorous simulation process, the combination of the actual values of angles of coupler links, corresponding to the optimized gait pattern can be achieved.
\end{abstract}

Keywords: - gait pattern, octopod, Klann's linkage, coupler angles $* * *$

\section{INTRODUCTION}

Robotics has been a center of fascination since 1970's and 80's. Being a part of Artificial Intelligence, robotics has undergone unimaginable development. Robotics has taken over everything from simple grippers to complex but superior humanoid robots.

This paper attempts to introduce to you a topic which lies on a similar line. Legged robots have been premeditated since last several decades. This project basically aims at replicating the gait pattern of an animal or insect leg. Leg is an absolutely precise alternative to the wheel, which may lead to undesirable results under rough terrains.Klann's Linkage, named after Joe Klann, was developed in 1994. Primarily, it was a 6 link mechanism consisting of a fixed frame, one crank, two rockers and two couplers. Kinematically, it can be categorized as 'Stephenson's type III linkage, ${ }^{[1]}$. (Classification of Stephenson's six-bar linkages depend upon the link which is chosen to be frame. ${ }^{[2]}$ )

Klann's linkage has been a fascinating topic for robotics enthusiasts. Since its invention in 1994, it has been implemented and studied worldwide by many scholars. The inventor, Joe Klann developed this linkage and used it for
Burmester curves which were used to design four bar linkages ${ }^{[3]}$.

But the main feature of Klann's linkage is that it closely resembles the shape of a leg of an insect like spider ${ }^{[3]}$. So primarily, Klann's linkage is used for locomotive applications. They are also used for applications which do not demand human intervention. Since the invention of Klann's linkage, walking robots having six-legs have been the choice of researchers and inventors. Walking robots are especially useful in rough terrains where wheels tend to become sloppy ${ }^{[4]}$. Number of walking robots have been designed using Klann's mechanism and the have been working in variety of environments and terrains very elegantly. Walking robots have excelled in variety of terrains. Although the difference in the capabilities of legged robots and humanoid robots is too large, legged robots have earned the importance because of their ease in maneuverability ${ }^{[5]}$.

\section{LITERATURE SURVEY}

Biomimetic robots have been an area of fascination for researchers. As a result, stupendous work has been done in the fields of wheeled robots, legged robots, humanoid robots, and what not. 
The pioneers in the field of 'legged robots' are dating back to 230 B.C. Greek Engineer Ctesibius developed the first moving linkage for locomotion which used pneumatics [6].

Around 1495, Leonardo da Vinci constructed the first anthromorphic robot [7]. It was made of wood and some parts were of leather and bronze. But wood made this robot weaker.

After da Vinci, the research slowed a bit down. But around 1738, Pierre JaquetDroze built a legged robot [6]. It consisted of many cams and over 400 parts, which made it a bit complex.

1850's, Russian Mathematician Chebyshev invented a kinematic linkage for the locomotion [6]. He also successfully presented its mathematical model which was a huge achievement in those days.

Throughout the $20^{\text {th }}$ century, huge work has been done in this area. But the main predicament with the existing legged robots is that differential turning generally becomes a tedious job. Students at MIT have been studying the variation of various kinematic parameters and studied the motion of legged robot ${ }^{[8]}$. Varying crank input, varying motor speed, varying link length ratios, are some of the exercises that people have dogged into. The effect of variation of coupler angles is an area where very less people have looked into. The main aim of this paper is to the effect of variation of angles of ternary links on the gait pattern of an octopod robot using Klann's linkage.

\section{OBJECTIVES}

- To establish a mathematical model for an octopod robot using Klann's linkage.

- To study the effect of variation of coupler angles on the gait pattern of Klann's linkage.

- To study the effect of variation of angle of leg link on the gait pattern of Klann's linkage.

- To achieve a peculiar combination of linkage parameters corresponding to an optimized gait pattern.

\section{KINEMATIC MODELLING}

As already stated, Klann's linkage is a kinematic linkage primarily used for replicating the leg of an insect or an animal. Klann's linkage is a 6-link mechanism having 7 joints and 1 degree of freedom. Type of joint used is 'revolute joint'. The following are the kinematics elements of Klann's mechanism.

a) Points $\mathrm{A}, \mathrm{C}$ and $\mathrm{F}$ represent the fixed frame.

b) Link $\mathrm{AB}$ is a crank.

c) Links CD and EF are rockers

d) Links BDG and EGH are couplers.

Here, the position analysis of Klann's mechanism has been presented by using 'Complex Algebra Method'.
Table 1: Notations used

\begin{tabular}{|l|l|}
\hline $\begin{array}{l}\text { Sr. } \\
\text { No. }\end{array}$ & Notations \\
\hline 1$)$ & $\begin{array}{l}\text { Co-ordinates of points :- } \\
\end{array}$ \\
$\mathrm{A} \equiv\left(a_{x}, a_{y}\right), \quad \mathrm{B} \equiv\left(b_{x}, b_{y}\right) \quad, \quad \mathrm{C} \equiv\left(c_{x}, c_{y}\right)$, \\
$\mathrm{D} \equiv\left(d_{x}, d_{y}\right), \mathrm{E} \equiv\left(e_{x}, e_{y}\right) \quad, \quad \mathrm{F} \equiv\left(f_{x}, f_{y}\right)$, \\
$\mathrm{G} \equiv\left(g_{x}, g_{y}\right), \mathrm{H} \equiv\left(h_{x}, h_{y}\right)$
\end{tabular}

\subsection{Loop Closure Equation for Loop ABCD}

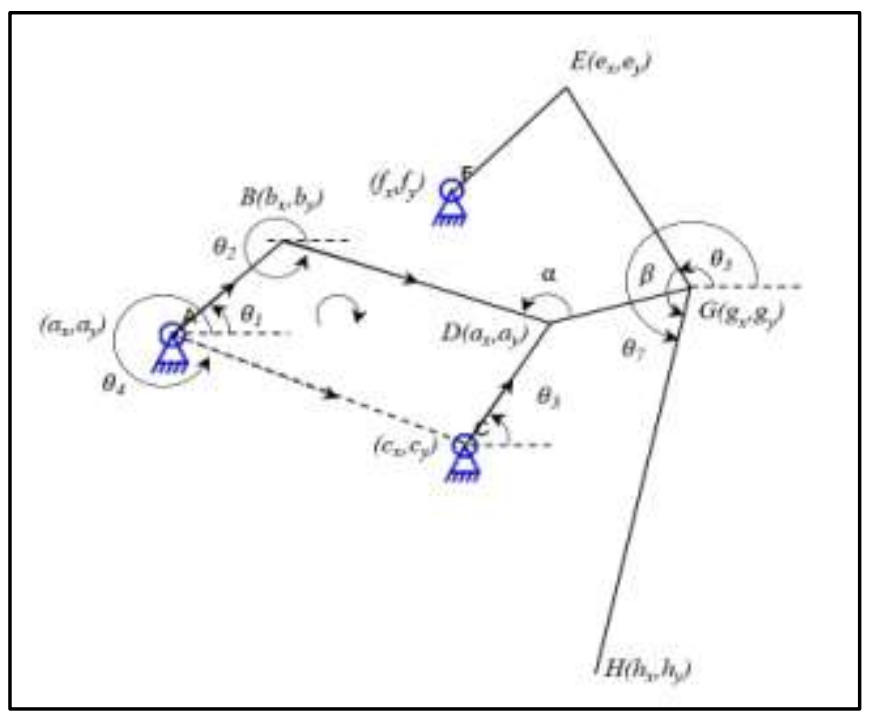

Fig 1: Kinematic representation of Klann's linkage

Considering a loop-closure equation for loop ABDC,

$$
\begin{gathered}
\overline{r_{1}}+\overline{r_{2}}-\overline{r_{3}}-\overline{r_{4}}=0 \ldots \ldots(1) \\
\therefore r_{1} e^{i \theta_{1}}+r_{2} e^{i \theta_{2}}-r_{3} e^{i \theta_{3}}-r_{4} e^{i \theta_{4}}=0 \\
\therefore r_{1}\left(\cos \theta_{1}+i \sin \theta_{1}\right)+r_{2}\left(\cos \theta_{2}+i \sin \theta_{2}\right)-r_{3}\left(\cos \theta_{3}+\right. \\
i \sin \theta 3-r 4 \cos \theta 4+i \sin \theta 4=0 \ldots(3)
\end{gathered}
$$

Using the trivial mathematical results and the technique of complex algebra, position analysis of any desired point can be performed.

\subsection{Locating the Co-ordinates of Point D}

Careful analytical treatment will show that the co-ordinates of the output point $\mathrm{D}$ are given by the equations:

And

$$
d_{x}=r_{1} \cos \theta_{1}+r_{2} \cos \theta_{2}=a \cos \theta_{1}+b \cos \theta_{2}
$$




$$
d_{y}=r_{1} \sin \theta_{1}+r_{2} \sin \theta_{2}=a \sin \theta_{1}+b \sin \theta_{2} \ldots .(4)
$$

\subsection{Position Analysis of Output Link GH}

Careful analytical treatment will show that the co-ordinates of the output point $\mathrm{H}$ are given by the equations:

$$
h_{x}=g_{x}+g \cos \left(\theta_{7}\right)
$$

And

$$
\begin{gathered}
h_{y}=g_{y}+g \sin \left(\theta_{7}\right)(5) \\
h_{x}=g_{x}+g \cos \left(\theta_{7}\right) \\
h_{y}=g_{y}+g \sin \left(\theta_{7}\right)
\end{gathered}
$$

\section{SIMULATION}

The primary intent of the simulation was to analyze the effect of variation of coupler angles on the gait pattern of the Klann's linkage. Accordingly, the the effect of variation of two coupler angles namely $\alpha$ and $\beta$ on the gait pattern was studied. The results of the simulation have been provided below:

\subsection{Effect of variation of $\beta$}

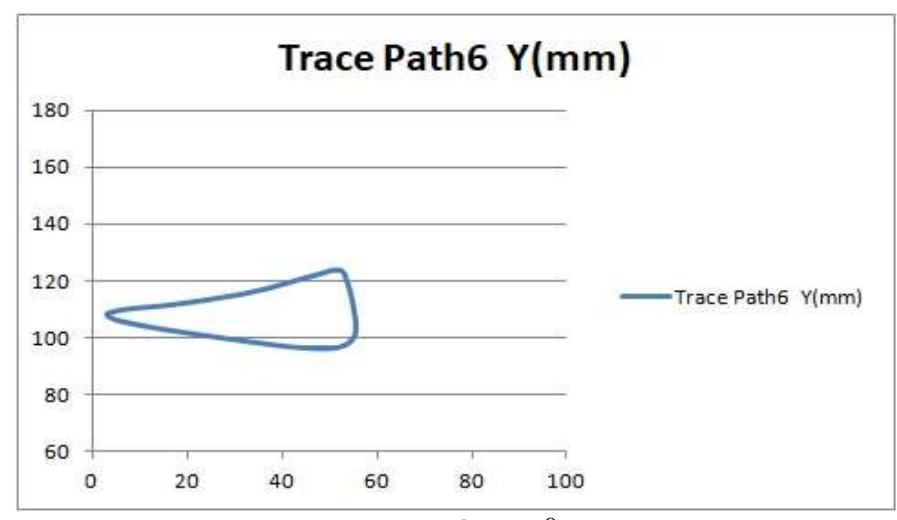

Graph 1: $\beta=125^{\circ}$

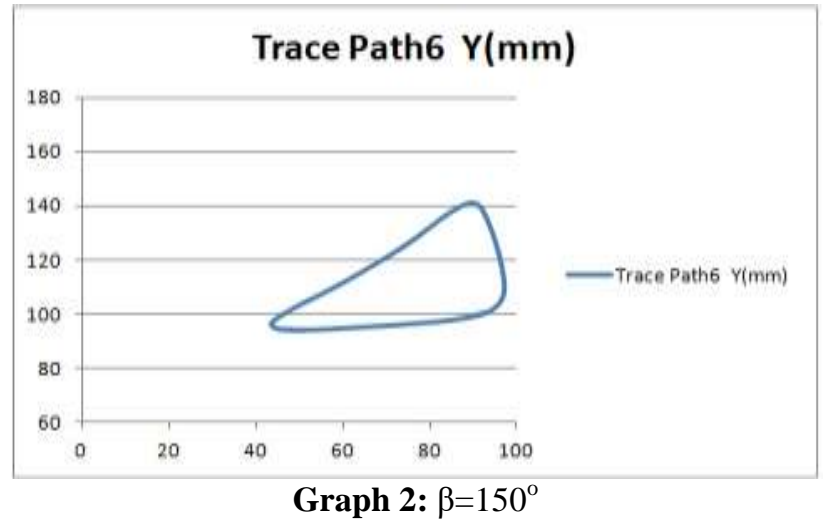

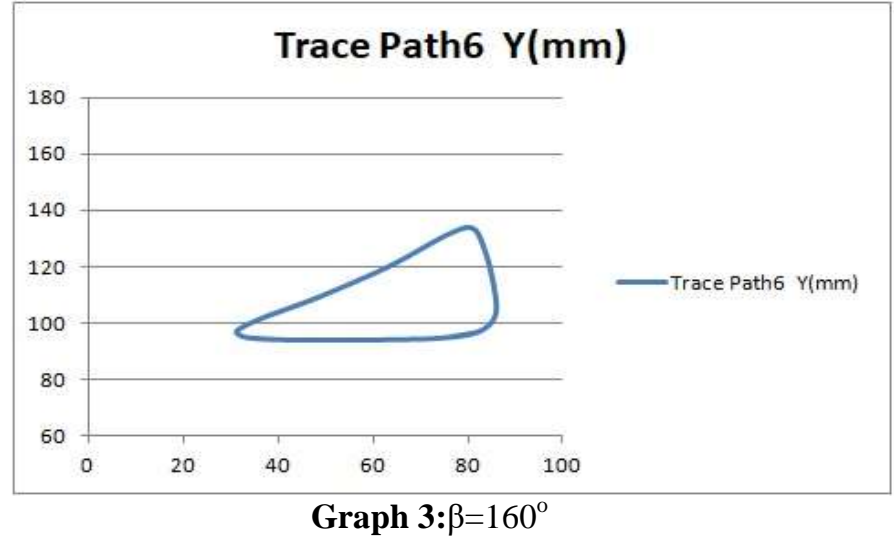

\subsection{Effect of variation of $\alpha$}
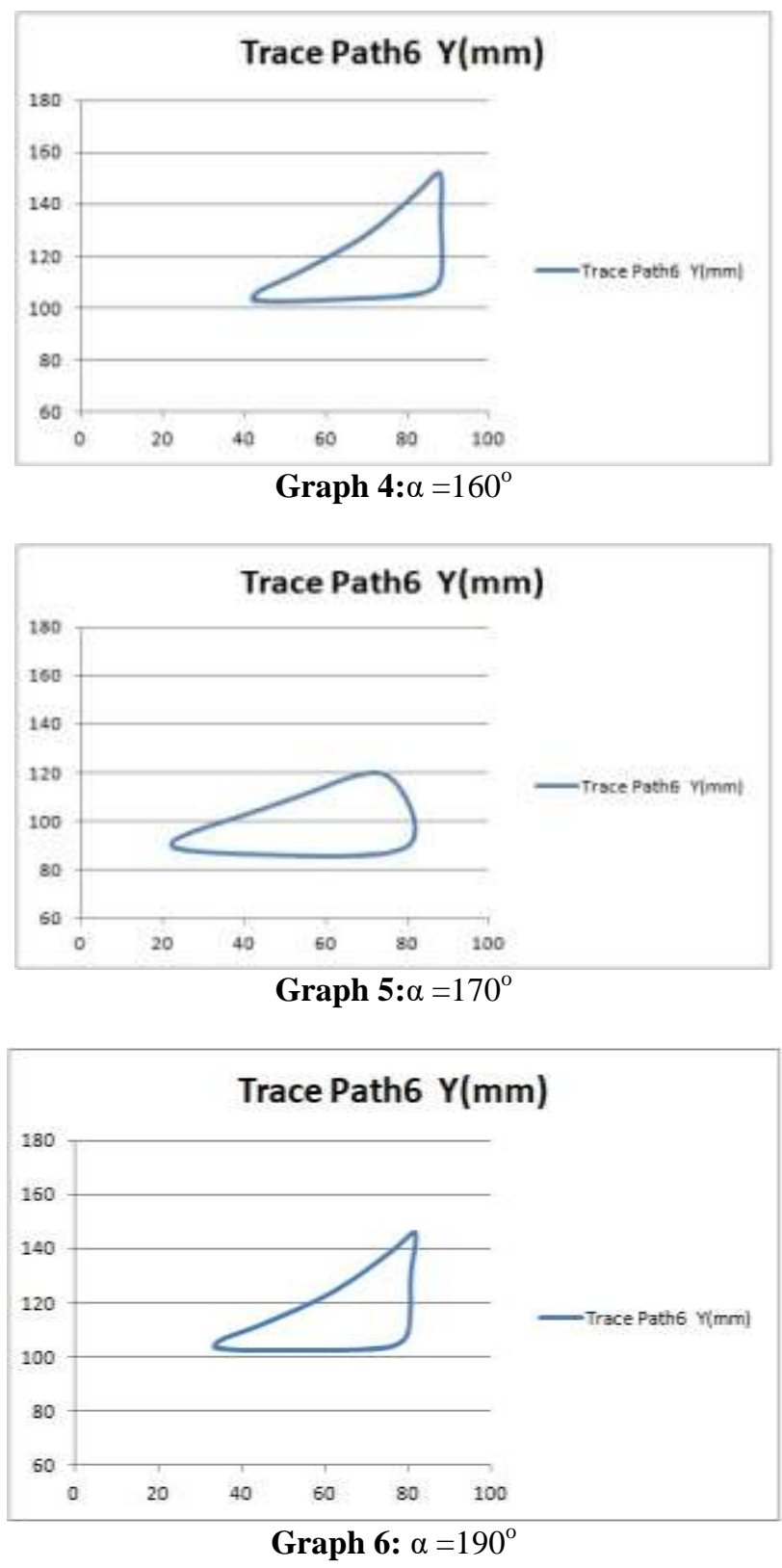


\subsection{Combination of $\alpha$ and $\beta$ for an Optimized Path}

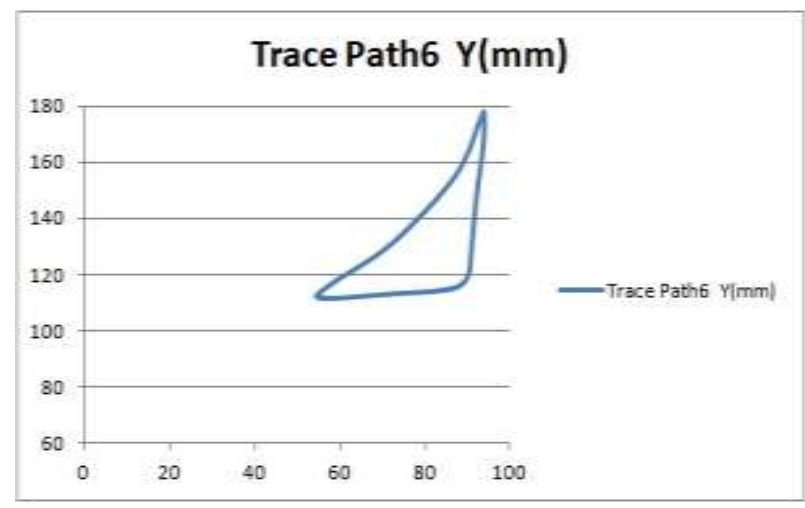

Graph 7: $\alpha=170^{\circ}$ and $\beta=143^{\circ}$

\section{PROTOTYPING}

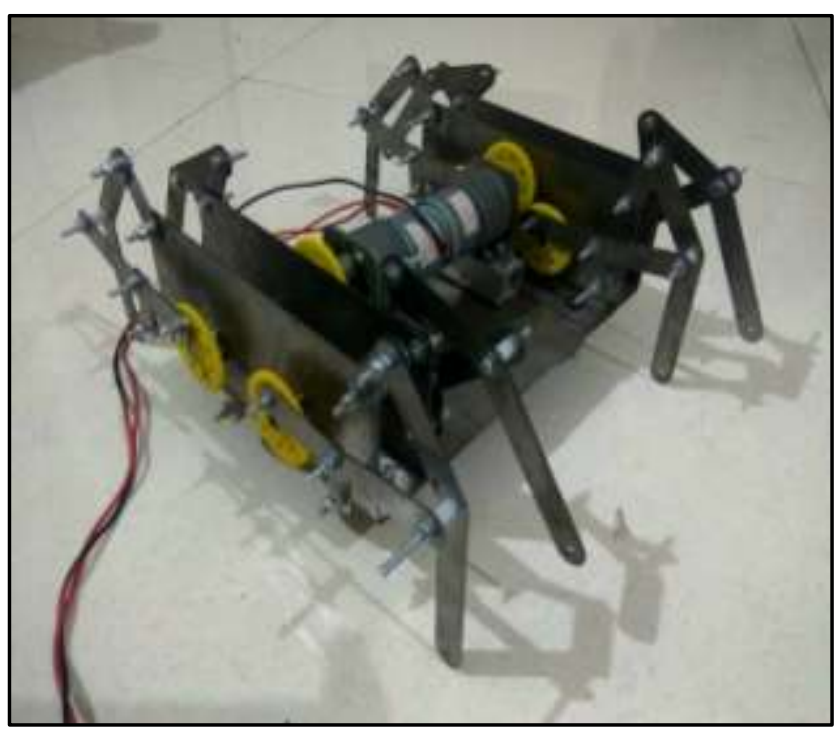

Picture 1: The prototype of the octopod spider robot

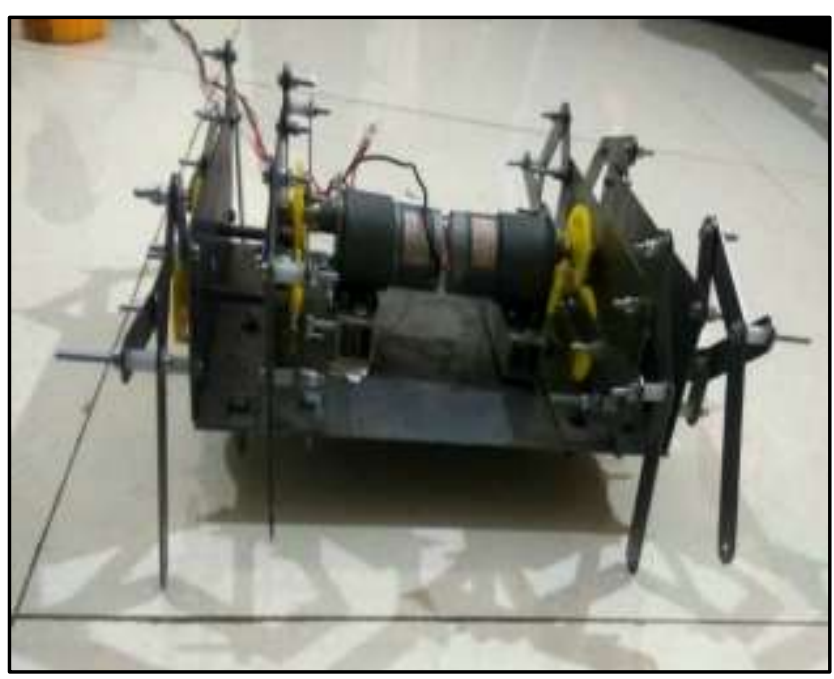

Picture 2: Arrangement of gears

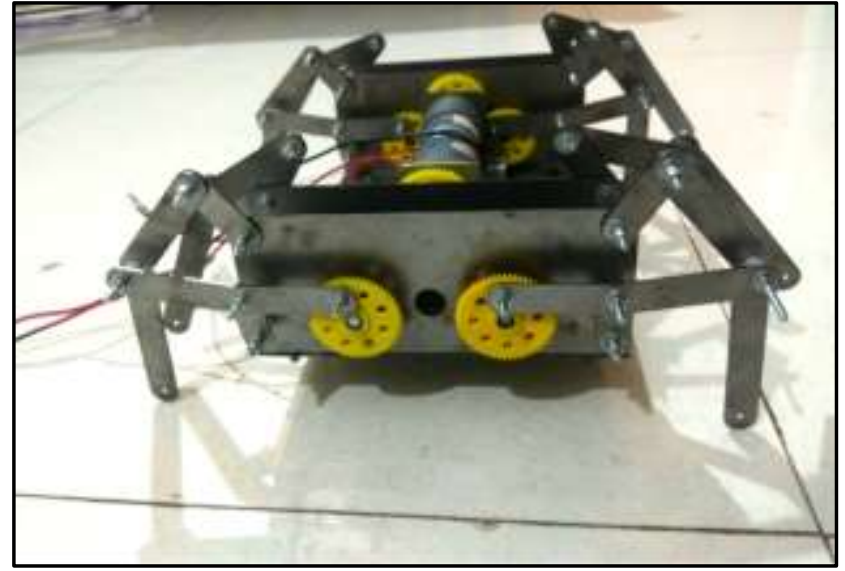

Picture 3: Arrangement of motors

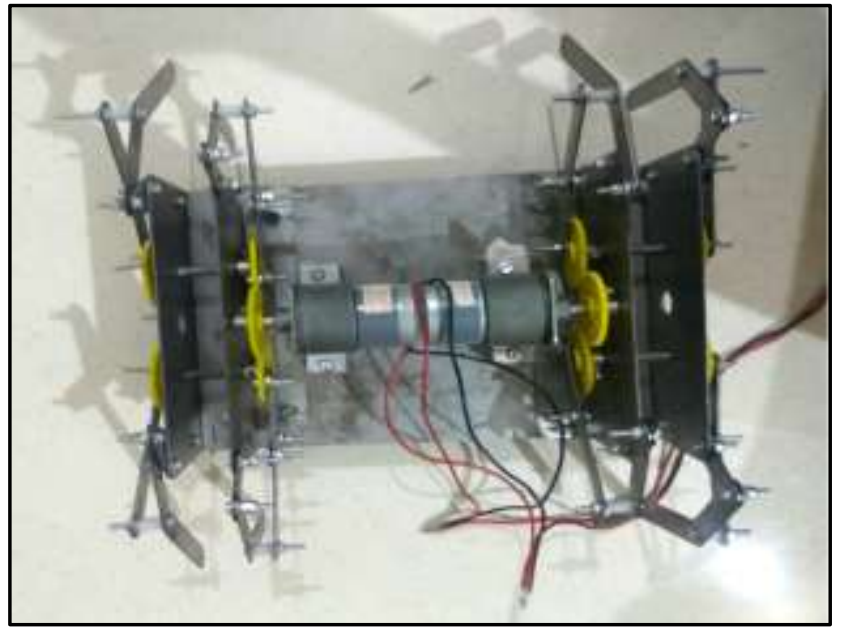

Picture 4: Top view of the assembly

\section{EXPERIMENTATION}

The octopod spider robot was made to undergo several tests and comprehensible observations have been collected from the experimental approach. The octopod spider robot was examined and the observations have been systematically tabulated.

As a basis for experimentation, the criteria selected was 'speed'. Well recorded experimental observations tabulated in the table given below provides a quantitative insight about the effect of variation of coupler angles. The purpose of experimentation is to experimentally verify the results of simulation and to obtained the most precise gait pattern by studying the variation of coupler angles.

Table 2: Observations obtained from experimentation

\begin{tabular}{|l|l|l|l|}
\hline $\begin{array}{l}\text { Coupler } \\
\text { angles } \\
\text { (in } \\
\text { degree) }\end{array}$ & $\begin{array}{l}\text { Distance(in } \\
\text { cm) }\end{array}$ & $\begin{array}{l}\text { Time(in } \\
\text { sec) }\end{array}$ & $\begin{array}{l}\text { speed(in } \\
\mathbf{c m} / \mathbf{s})\end{array}$ \\
\hline \multirow{3}{*}{$160^{\circ}$} & 60 & 36.32 & 1.65198 \\
\cline { 2 - 4 } & 80 & 48.89 & 1.63632 \\
\cline { 2 - 4 } & 100 & 61.21 & 1.63371 \\
\hline & 60 & 27.19 & 2.20669 \\
\hline
\end{tabular}




\begin{tabular}{|l|l|l|l|}
\hline $170^{\circ}$ & 80 & 37.03 & 2.16041 \\
\cline { 2 - 4 } & 100 & 46.77 & 2.13812 \\
\hline \multirow{3}{*}{$190^{\circ}$} & 60 & 31.70 & 1.89274 \\
\cline { 2 - 4 } & 80 & 43.49 & 1.83950 \\
\cline { 2 - 4 } & 100 & 54.81 & 1.8244 \\
\hline
\end{tabular}

\section{RESULTS AND DISCUSSION}

After testing the octopod spider robot and analyzing every set of observations, the results of the experiments are giving a comprehensible insight into the intended relationship between coupler angles and gait pattern and thus, resolving the very purpose of the experimentation. Moreover, the observations of the experiments are found to be in good agreement with the theoretical relationship as well as the results of the simulations.

a) The relationship between the time required to cover a defined distance for different $\alpha$ is as follows: -

b) The relationship between the speeds over a defined distance corresponding to different $\alpha$ is as follows: -

This is in total agreement with the results obtained through simulations.

\section{CONCLUSION}

- A comprehensible mathematical model was established for a robot using Klann's linkage.

- The variation of coupler angles on gait pattern of Klann's linkage results in the change of shape of the path.

- The variation of angle of leg link on the gait pattern of Klann's linkage results in the change of slope of the path.

A combination of $\alpha=170^{\circ}$ and $\beta=143^{\circ}$ results in the precise gait pattern.

\section{FUTURE SCOPE}

- The spider robot can be facilitated with differential turning.

- The spider robot can be controlled wirelessly by making use of Arduino circuitry and programming.

- The walking speed of the spider robot can be enhanced by using the motors having higher speeds.

- The spider robot can be made to climb the slopes by manipulating the gait pattern.

- The spider robot can be made to climb the slopes by manipulating the gait pattern.

\section{REFERENCES}

[1] Eugenie Carol Scott, 'Evolution Vs. Creationism : An Introduction', 2009, California, University of California Press.
[2] Michael Nosonovsky, Bharat Bhushan, 'Green Tribology: Biomimetics, Energy Conservation and Sustainability', Springer Verlag-Berlin Heidelberg, Springer International Publishing.

[3] Harry Henderson, 'Artificial Intelligence : Mirrors for the Mind', 2007, Chelsea House, Chelsea-InfoBase Publishing.

[4] Kevin Russell, Qiong Shen, Raj S. Sodhi, 'Mechanism Design : Visual and Programmable Approaches', 2013, Boca Raton, CRC Press, Taylor and Francis Group.

[5] E.A. Dijksman, 'Motion Geometry of Mechanisms', 1976, Cambridge University Press, Cambridge.

[6] Learning SOLIDWORKS 2016, Randy Shih, 2015, SDC Publications, United States of America.

[7] Bruno Siciliano, Oussama Khatib, 'Springer Handbook of Robotics', 2008, Springer-Verlag Berlin Heidelberg, Springer International Publishing.RolandSiegwart, Illah R. Nourbakhsh, Introduction to Autonomous Mobile Robots, Second Edition, 2011, The MIT Press, Cambridge.

[8] Kenzo Nonami, Ranjit Kumar Barai, Addie, 'Hydraulically Actuated Hexapod Robots : Design, Implementation and Control', 2014, Japan, Springer International Publishing.

[9] Millie Pant, Kusum Deep, Atulya Nagar, Jagdish Chand Bansal, 'Proceedings of the Third International Conference on soft Computing for Problem Solving', Volume I, 2014, India, Springer International Publishing.

[10] Alexander Shkolnik, Michael Levashov, Ian R Manchester and Russ Tedrake, 'Boundings on Rough Terrain with the Little Dog Robot', 2010, The International Journal of Robotics Research, SAGE Publications.

\section{BIOGRAPHIES}

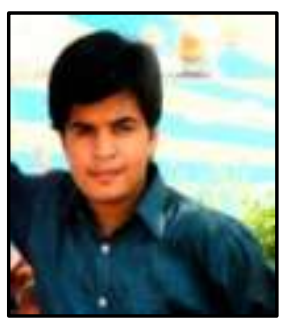

Pratik Walimbe is currently pursuing his Bachelor of Technology (B.Tech) in Mechanical Engineering at the Department of Mechanical Engineering,Vishwakarma Institute of Technology, Pune, India. His research interests include Kinematics and Dynamics of Machinery, Optimization, Biomimetics, Energy.

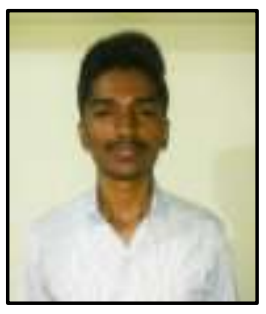

Akash Gunjal is currently pursuing his Bachelor of Technology (B.Tech) in Mechanical Engineering at the Department of Mechanical Engineering,Vishwakarma Institute of Technology, Pune, India. His research interests include Design for Sustainability and Biomimetics. 


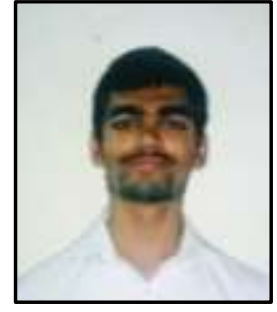

PranavkumarNehrotra is currently pursuing his Bachelor of Technology (B.Tech) in Mechanical Engineering at the Department of Mechanical Engineering,Vishwakarma Institute of Technology, Pune, India. His research interests include Automobiles, Automation, Biomimetics.

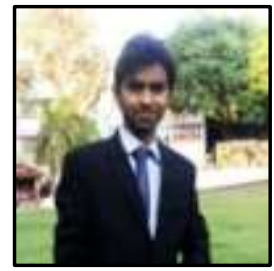

Manish Chaudhari is currently pursuing his Bachelor of Technology (B.Tech) in Mechanical Engineering at the Department of Mechanical Engineering,Vishwakarma Institute of Technology, Pune, India. His research interests include Designing, Robotics, Simulation and Analysis, Manufacturing Processes. 